\title{
Review of SDM/WDM technology and its application in data transmission
}

\author{
J. R. Warnes-Lora ${ }^{a}$, L. J. Quintero-Rodríguez ${ }^{a}$, B. Tshibangu-Mbuebue ${ }^{a}$, A. García-Juárez $^{b}$, and I. E. Zaldívar-Huerta $^{a, *}$ \\ ${ }^{a}$ Depto. de Electrónica, Instituto Nacional de Astrofísica, Óptica y Electrónica, \\ Calle Luis Enrique Erro No.1, Tonantzintla, Puebla 72840, México. \\ *e-mail: zaldivar@inaoep.mx \\ ${ }^{b}$ Depto. de Investigación en Física, Universidad de Sonora, \\ Blvd. Luis Encinas y Rosales S/N, Hermosillo, Sonora 83000, México.
}

Received 10 December 2020; accepted 20 January 2020

\begin{abstract}
In this paper, we present a review focused on Space Division Multiplexing (SDM) technology. A summary of the most recent works on innovation in the efficiency of SDM as well as its application in data transmission is described. SDM is a technology that has improved the capacity of optical communication systems. Combined with other optical technologies has significantly increased the capacity of information that is sent through an optical fiber.
\end{abstract}

Keywords: Space division multiplexing; wavelength division multiplexing; few-mode fiber; photonic lantern.

PACS: $42.79 . \mathrm{Sz}$

\section{Introduction}

Currently, most users are demanding services of high quality and high speed such as HDTV, Internet, etc. Face to this demand, optical communication systems have demonstrated to be a good candidate to deliver these types of services. In order to exploit the capacity of the optical fiber to distribute information to a big number of users, several techniques have been developed. In this regard, Wavelength Division Multiplexing (WDM), Time Division Multiplexing (TDM), and Space Division Multiplexing (SDM) have emerged as excellent technological alternative. In this sense, Fig. 1 shows a graphical evolution as well as a tendency of these techniques for the next years [1].

From the three techniques (WDM, TDM, and SDM) previously mentioned, each of them possesses its characteristics.

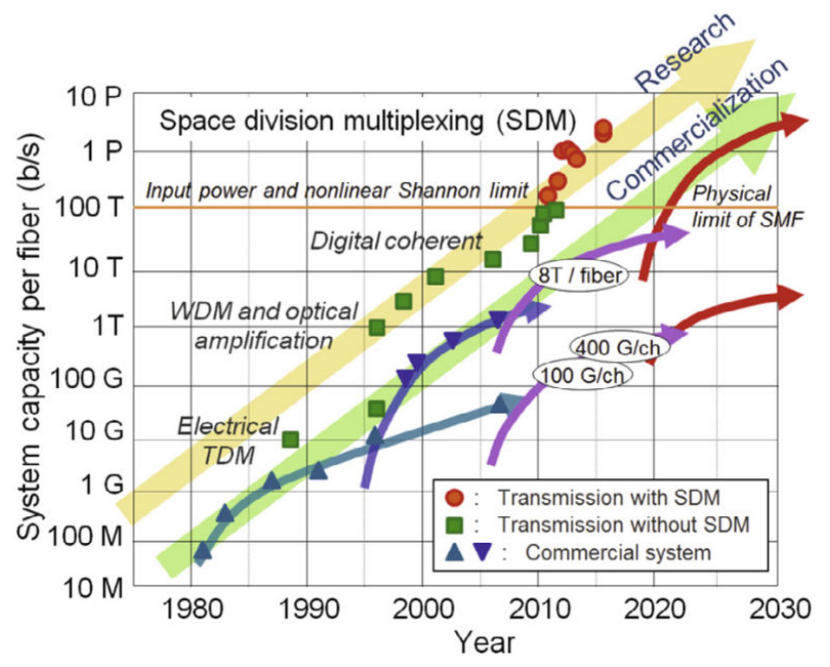

FIGURE 1. System capacity per fiber in optical communication systems [1].
As is illustrated in Fig. 1, in the early 80s, TDM technology was used to transmit a considerable amount of information through optical fibers; however, with the advent of WDM, this technology became obsolete. Currently, WDM is capable to reach the data rate of Terabits per second. On another side, SDM is a technology that emerged in the early 2010 s [2]. This technology takes advantage of the characteristics of the Photonic Lantern (PL) device [3-9]. Unlike WDM, which uses different wavelengths to transmit information, SDM employs linearly polarized modes (LP or LP modes) for transmission. SDM in combination with Multicore Optical Fibers (MCF) [10], Multimode Optical Fibers (MMF) [10,11], and Few Mode Fiber (FMF) [12] have made it possible to reach transmission rates in the order of Petabytes per second [1], as is depicted in Fig. 1.

Given the scenario above described, the goal of this paper is to summarize a review of the most recent research about SDM/WDM techniques. This work is composed of two sections. The first section is focused on showing the new contributions in the areas of PL, MCF, and FMF for their application in WDM/SDM. The second section is devoted to presenting works related to transmission rates using WDM and SDM multiplexing techniques as well as the integration of components such as PL, MCF, and FMF.

\section{SDM Technology and its application in data transmission}

\subsection{SDM technology}

In the last few years, MMF has been used in short-distance applications such as Local Area Networks (LAN), indoor networks, or Data-Center Networks (DCN) due to its low cost and easy operation. However, face with the enormous and fast-growth of internet traffic and the development of some applications such as big data and clouding computing, it is 


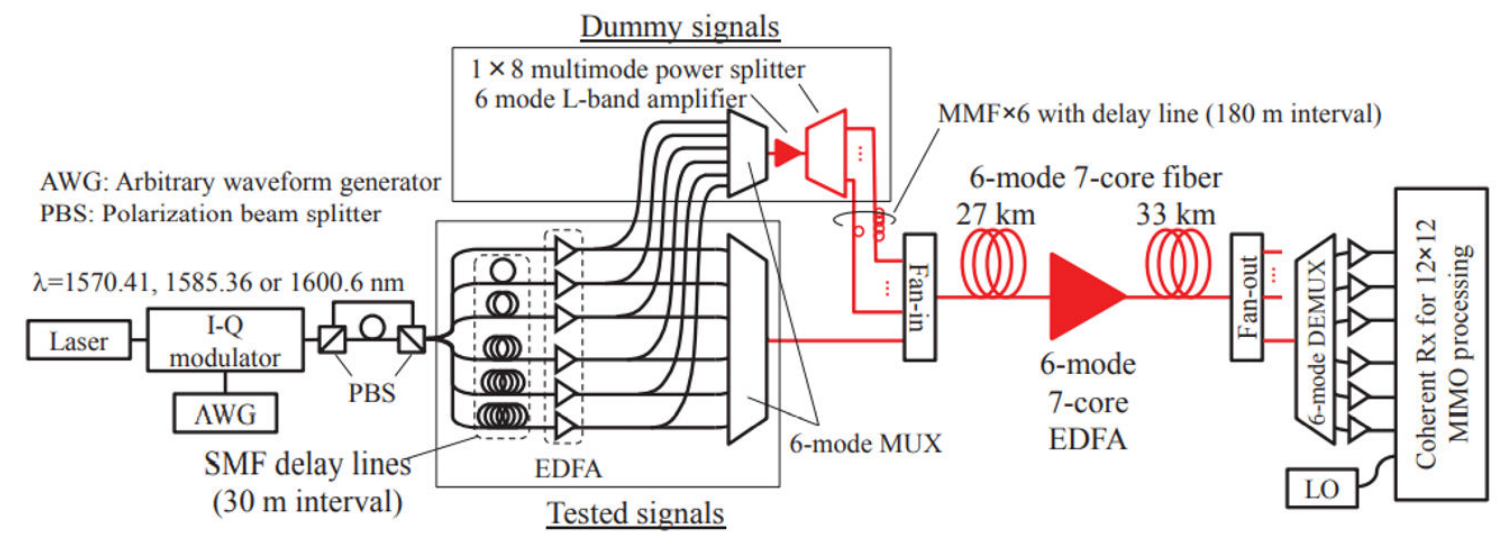

FIGURE 2 . Experimental configuration for in-line amplified 6-mode 7-core multicore fiber transmission linewidth a 6M-7C EDFA [18].

necessary to increase the transmission capacity of the MMF links in order to satisfy the bandwidth demand. Nevertheless, the transmission capacity of the links that use MMF is limited by modal dispersion. Fortunately, several promising approaches have been proposed to increase the capacity of transmission in the MMF links. For instance, electrical dispersion compensation (EDC) [13], mode-selective excitation with a spatial light modulator (SLM) [14], filtering out higher-order modes by using single-mode fibers (SMF) or photonic crystal fibers (PCF) $[15,16]$.

Lately, in Ref. [17], a weakly-coupled mode division multiplexing over conventional multi-mode fiber (MMF) with intensity modulation and direct detection scheme was proposed to overcome the modal bandwidth limitation of MMF. An MMF Corning OM3 parabolic refractive index profile that supports about 36 modes at the wavelength of $1550 \mathrm{~nm}$ was used. This work highlights its successful operation for high-speed optical interconnections and its increase in transmission capacity for short-reach systems and networks. In Ref. [18], a high-density few-mode multicore fiber (FM-MCF) amplifier for energy-efficient spacedivision multiplexing (SDM) transmission with the highest spatial density was accomplished. Here, a cladding-pumped 6-mode-7-core (6M-7C) L-band EDFA with a power consumption reduction factor of 0.36 and an in-line amplified SDM data transmission over an FM-MCF was experimentally demonstrated. Remarkably the power reduction factor achieved in this work, which is considered as a new achievement for the cladding-pumped MCF amplifiers, reported up to date. Figure 2 illustrates the experimental configuration for the simultaneously amplified 6-mode 7-core fiber transmission experiment. Three signals were generated, multiplexed, and divided into six parts in order to obtain six signals to be multiplexed by a 6 -mode multiplexer and injected into a test core. The other 6-mode multiplexed signals were injected into the next 6 cores of the MCF and used as dummy channels. As a result, propagation losses of $0.21 \mathrm{~dB} / \mathrm{km}$, differential mode delay of $0.50 \mathrm{~ns} / \mathrm{km}$, and core-to-core crosstalk of the $6 \mathrm{M}-7 \mathrm{C}$ fiber in the L-band of $-21 \mathrm{~dB} / 100 \mathrm{~km}$ for all modes were obtained, respectively.
Regarding SDM transmission technology. In Ref. [19] is summarized the latest advances obtained to increase the power efficiency in SDM as well as in multicore amplifiers. For SDM technology, parallelization, signal allocation, and routing algorithms were considered alternative approaches to increase the transmitted capacity and maximize power efficiency. Also, multicore amplifiers are reviewed as an important part of transmission systems where uncoupled core multicore fiber amplification, coupled core multicore fiber amplification technologies allow reduce the power consumption of amplification. All this causes more tolerance to nonlinear distortion, increasing in this way the power efficiency in SDM transmission systems.

In Ref. [20], SDM transmission schemes over FMFs and multicore (MC) FMFs using mode division multiplexing (MDM) technology to achieve long-haul high-capacity SDM transmission using mode multiplexing were reviewed. Here, it is also highlighted linear propagation effects, including differential mode delay (DMD) and mode-dependent loss (MDL), as performance-limiting factors in long-haul MDM transmission.

Another proposed technique for WDM/SDM transmission with ultra-high aggregated spectral efficiency was demonstrated in Ref. [21]. A record-high spectral efficiency of $297.8 \mathrm{bit} / \mathrm{s} / \mathrm{Hz}$ for an SM-MCF transmission system was reached by using modulation format of PS-PDM-1024-QAM and soft-decision forward error correction (SD-FEC) with adaptive information rates for different spectral and spatial channels. Figure 3a) shows the experimental configuration proposed for the WDM/SDM transmission of PS PDM-1024QAM channels. Furthermore, the generated WDM channels by using this configuration with a $12.5 \mathrm{GHz}$ grid or a $25 \mathrm{GHz}$ grid are illustrated in Fig. 3b) and c), respectively. The heterogeneous single-mode 30-core fiber employed in the experimental configuration is depicted in Fig. 3d). It can be observed four different types of cores, which generate phase mismatch between neighboring cores, emerging in a high-density core arrangement with low cross-talk lower than $-50 \mathrm{~dB}$ after $9.6 \mathrm{~km}$ transmission line. As a consequence, the 


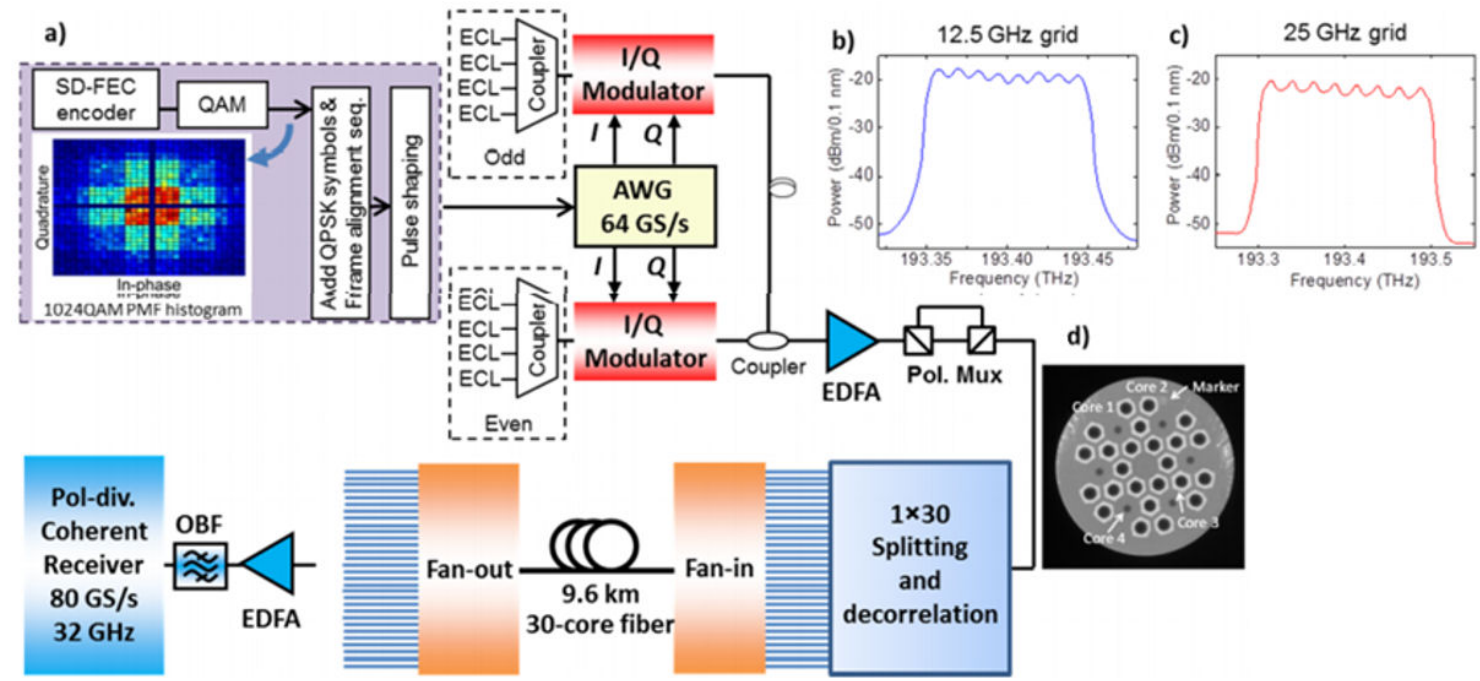

FIGURE 3. a) Experimental configuration for the WDM/SDM transmission of PS PDM-1024-QAM channels over a 30-core fiber; b) and c) optical spectra of the WDM channels after the 30-core fiber transmission; and d) cross-sectional view of the 30-core fiber [21].

spectral efficiency of $297.82 \mathrm{bit} / \mathrm{s} / \mathrm{Hz}$ and an aggregated net data rate per frequency channel of $3.72 \mathrm{Tbit} / \mathrm{s}$ were obtained in this work.

In Ref. [22], the authors present a 7-core 6-mode photonic lantern (PL) capable of selectively exciting LP01, LP11a, LP11b, LP21a, LP21b, and LP02 modes in each of the seven cores of a PL arranged in a hexagonal lattice. In order to obtain the selectivity of modes in the device, optical fibers of different diameters are used to have different propagation constants. Figure 4 shows how the authors coupled the PL to an FMF and shows the profile modes at the output of the FMF. This device can be tailored to be integrated with existing few-mode multicore fibers for high-density spacedivision multiplexing and is expected to be scalable to higher core count while maintaining compactness and fabrication repeatability by using microstructured glass templates.

In Ref. [23], the authors propose a high-selectivity photonic lantern demultiplexer for weakly-coupled mode group

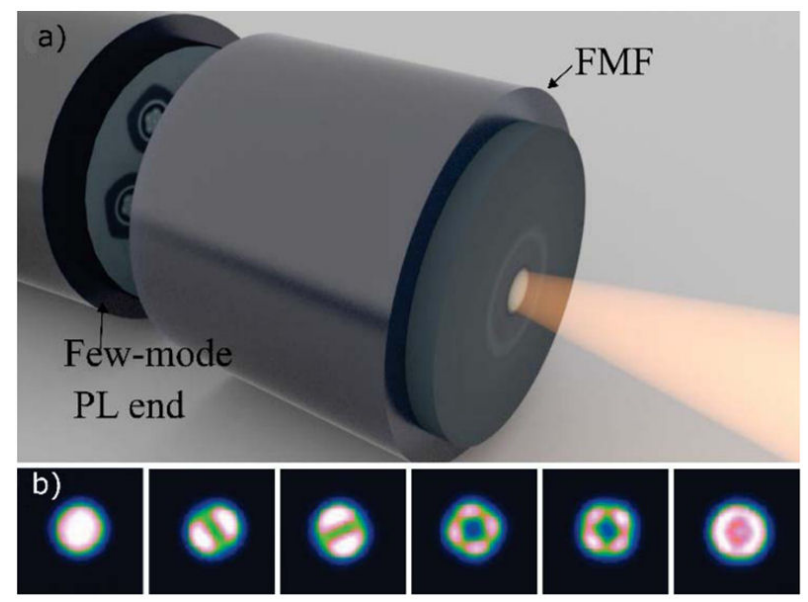

FIgURE 4. a) Central core of the few-mode multicore PL with a FMF b) Mode profile [22]. demultiplexing over MMF. Figure 5 shows the proposed structure, which is composed of three stages. At the input port, the MMF is coupled with the Mode-selective photonic lantern (MSPL). Then the PL is used to demultiplex all the LP modes with high selectivity. The main objective of this work was to demultiplex certain groups of LP modes in such a way that each PL in the final part of the scheme uses different core diameters to support different LP modes. The proposed scheme can also be combined with other optimization schemes and extended to demultiplex more groups of modes.

In Ref. [24], the authors use a PL in an external modulation system to distribute filtered microwave signals. For this goal, three modes of the PL (LP01, LP11a, and LP11b) were employed for the transmission of the microwave signal of $1.92 \mathrm{GHz}$ using the setup illustrated in Fig. 6.

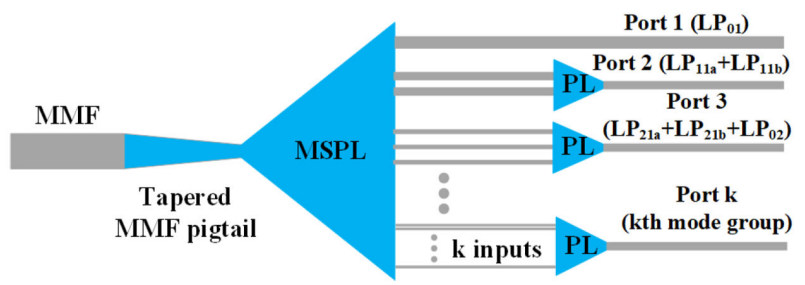

FIGURE 5. Schematic diagram of the mode group demultiplexer [23].

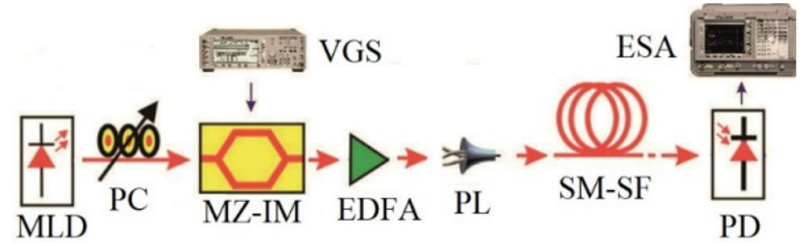

FIGURE 6. Experimental set up used to test the PL [24]. 


\subsection{Application in data transmission}

The theory published in Ref. [25] shows that the technological evolution of MCFs allowed them to perform very interesting optical SDM telecommunication systems characterized by a low level of inter-core crosstalk, a high number of cores, and a good transmission through long-haul links. As an essential component in realizing the integrated MCF, the multicore amplifiers used in the implementation of the multicore link are erbium-ytterbium-doped fiber amplifiers (MCEYDFAs), whose diagram is presented in Fig. 7.

In this work, the integrated multicore link (IML) used in the multicore fiber system to analyze performance transmission is constituted by a pair of 7-core MC-EYDFA, the fan-in and fan-out devices, and a $60 \mathrm{~km}$ of homogeneous trench-assisted 7-core MCF as exhibited in Fig. 8. It is shown transmission the achievability of 15 WDM channels per core signal test through $720 \mathrm{~km}$, which is constituted by a 200 Gbit/s 16-QAM signal and 14 de-correlated $100 \mathrm{Gbit} / \mathrm{s}$ QPSK neighboring channels. Considering a $50 \mathrm{GHz}$ channel grid in the range of $1558.58 \mathrm{~nm}$ to $1564.27 \mathrm{~nm}$, a performance of each MCF core such as the transmission distance, the intercore crosstalk and gain of MC-EYDFA, the insertion loss, and the bit-error rate (BER) and optical-signal-to-noise ratio (OSNR) measurement, was evaluated.

In Ref. [26], the authors present an architecture SDMWDM-PON that performs intensity modulation/direct detection (IM/DD) and uses low-crosstalk MCF link in the absence of digital signal processing (DSP). Figure 9 exhibits the experimental setup using a $2 \mathrm{~km}$ 7-core fiber: three outer cores for upstream (US), three distinct outer cores for downstream (DS), and the central inner core for light source transmission. The light travels from the optical line terminal (OLT) to the optical network unit (ONU), thereby establishing colorless

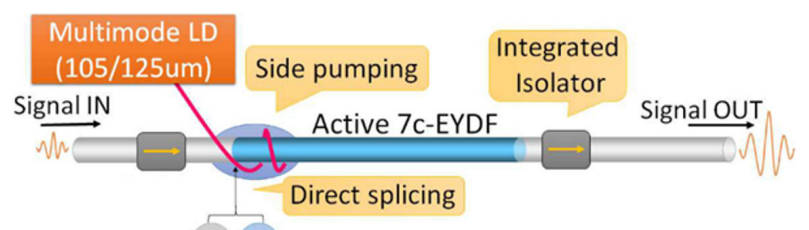

Passive 7c-MCF $\because:$ Active 7c-MCF

FIGURE 7. Internal structural diagram of an MC-EYDFA. The isolator position will depend on the specific amplifier role in its respective application [25].

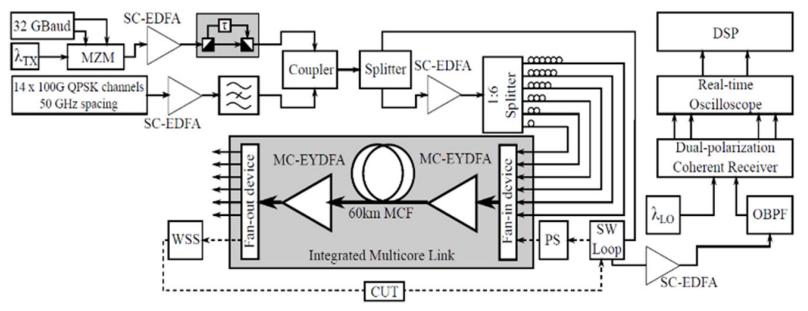

FIGURE 8. Experimental setup of the multicore fiber system [25].

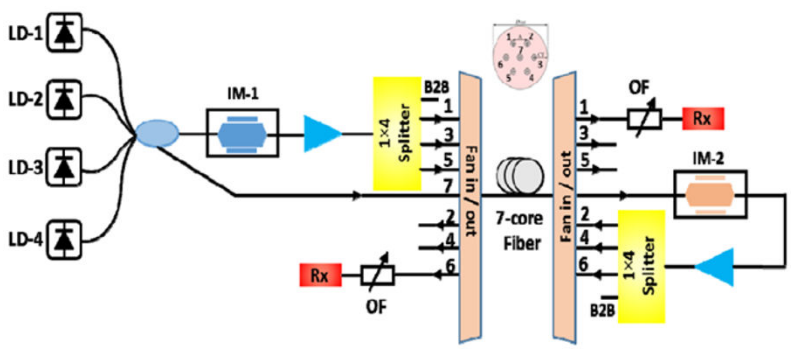

FIGURE 9. Experimental setup for SDM-WDM-PON system. (IM: intensity modulator, B2B: back to back) [26].

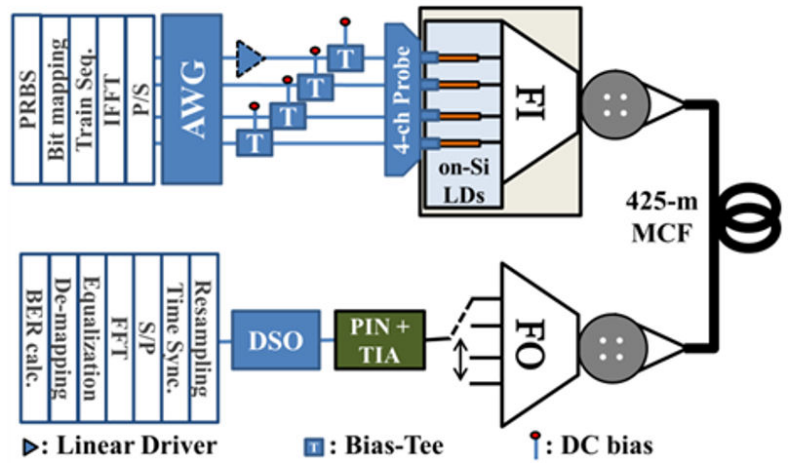

FIGURE 10. Experimental setup for DMT signals process. (PRBS: pseudo-random bit sequence; AWG: Arbitrary Waveform Generator; LD: Laser Diode; MCF: Multi-Core Fiber; (I)FFT: (Inverse) Fast-Fourier Transform; TIA: Trans-Impedance Amplifier; FI/FO: Fan-In/Out; DSO: Digital Sampling Oscilloscope; P/S (S/P): Serial-to-Parallel conversion; PIN: p-i-n photodiode) [27].
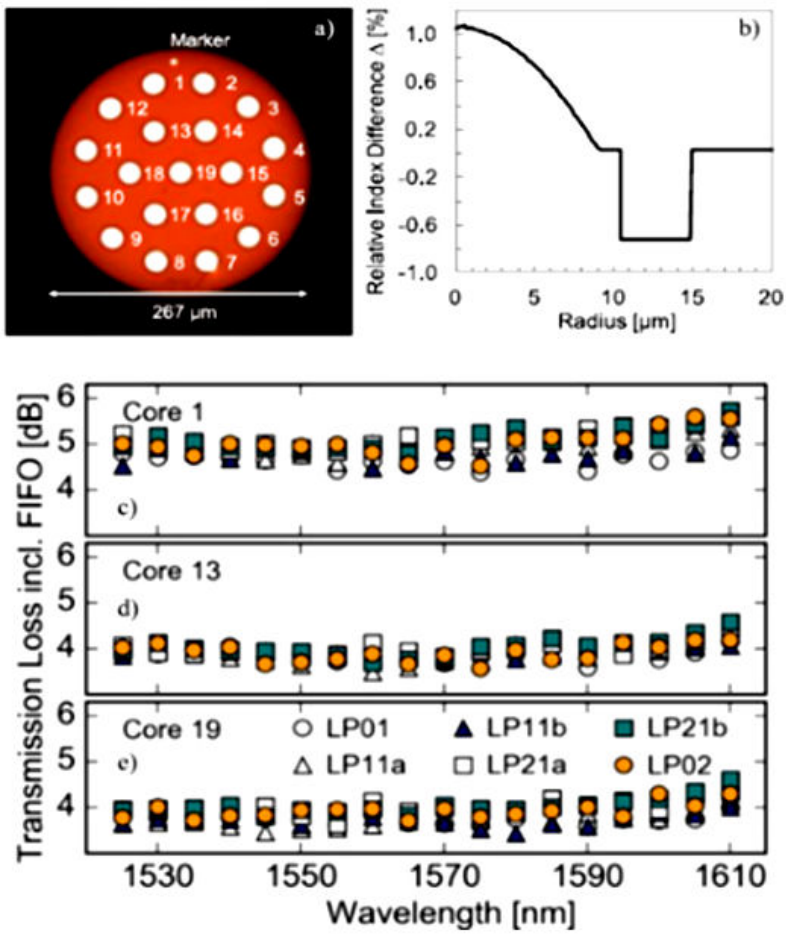

FIGURE 11. a) Cross section of the fabricated fiber. b) Estimated refractive index profile. c)d)e) Transmission loss including FIFO at cores \#1, 13, and 19 [28]. 


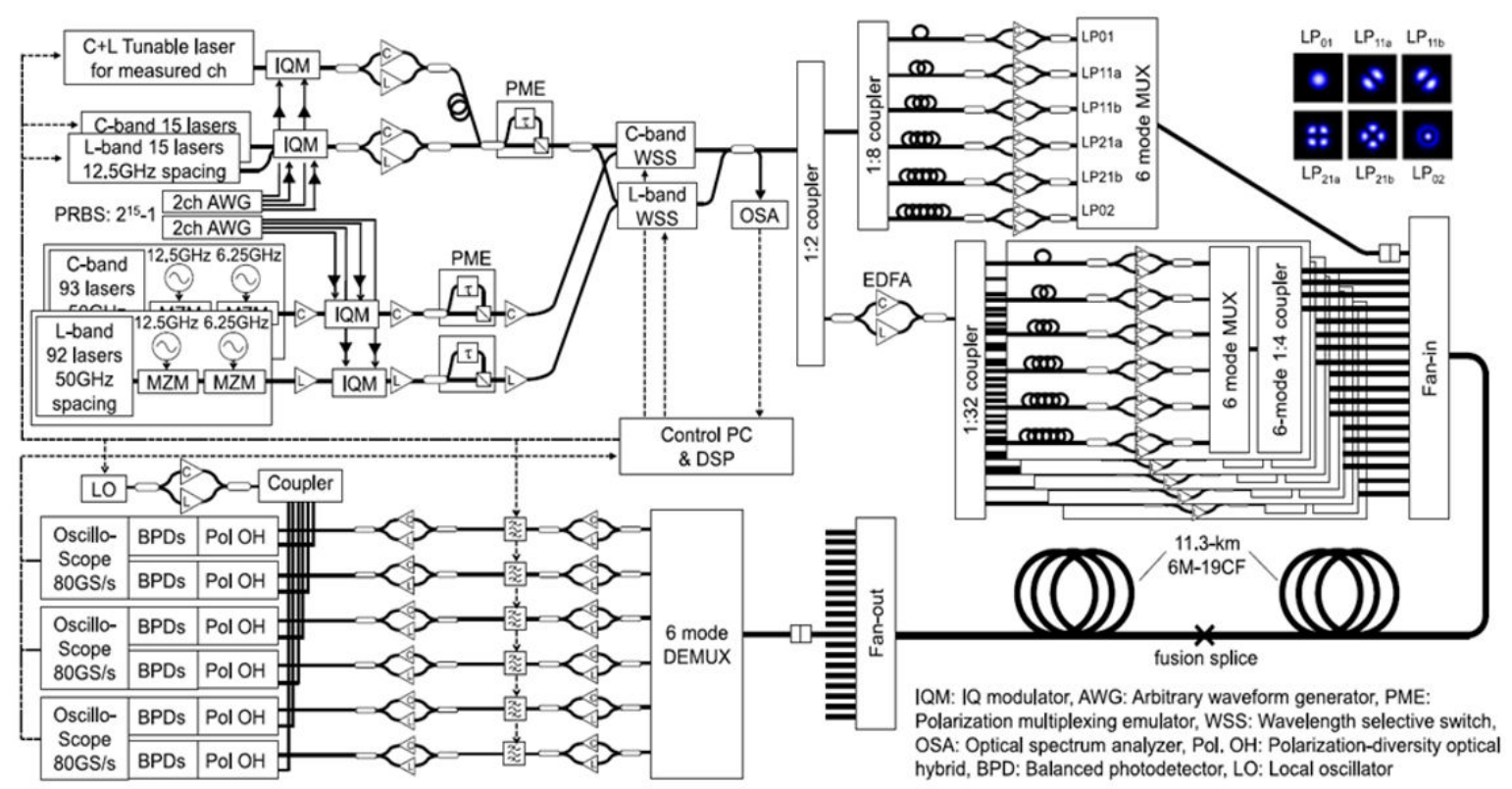

FIGURE 12. Experimental setup for the 10.16-peta-bit/s SDM/WDM transmission experiment [28].

and laserless ONUs and at the same time permitting the reduction of the cost and the facility of wavelength management. This experiment is remarkable because it provides $300 / 120 \mathrm{~Gb} / \mathrm{s}$ transmission capacity for DS/US (4 wavelengths, 3 cores, $25 / 10 \mathrm{~Gb} / \mathrm{s}$ per wavelength).

Under the same approach, in Ref. [27] is presented another promising technique with advantages such as low cost, low consumption of power, and small size by using transmitters multi-channel based on directly-modulated lasers (DMLs). Since WDM and SDM transmitters perform a $100 \mathrm{Gbit} / \mathrm{s}$ transmission by a single DML-om-Si, and a discrete multi-tone (DMT) or pulse-amplitude modulation (PAM) modulation, has been demonstrated. Even more, Ref. [27] shows a reliable transmission achieved over $425 \mathrm{~m}$ of MCF by a close-packed, SDM-scalable, MCF-pluggable, and power-efficient 4-channel SDM transmitter based on (DMT); and a $1.3 \mu \mathrm{m}$ membrane DML-array-on-Si. The arrangement illustrated in Fig. 10 was used to demonstrate experimentally that driving at the same time both lasers, and considering a $20 \%$ overhead SD-FEC limit of $2.0 \times 10^{-2}$, preFEC $400 \mathrm{~Gb} / \mathrm{s}(100 \mathrm{~Gb} / \mathrm{s} /$ channel, $320 \mathrm{~Gb} / \mathrm{s}$ net $)$ transmission is achieved through the $425 \mathrm{~m} \mathrm{MCF}$, noting that the total power required for driving the lasers was $102.5 \mathrm{~mW}$ (six times lower than that of conventional $1.3 \mu \mathrm{m}$ DMLs). However, the numerical simulation ensured the suitability of the system through an MCF link of $10 \mathrm{~km}$.

Subsequently, in Ref. [28], another interesting transmission technique that allows increases the capacity of transmission obtained in SMF is the SDM which uses MCF and FMF. Highlighting that with the compound or hybrid approach, the few-mode multi-core fibers (FM-MCFs) supported an experimental ultra-dense transmission with a spatial multiplicity of 114 was achieved by a 6-mode 19-core fiber (6M-19CF).
According to the maximum capacity of about $100 \mathrm{Tbit} / \mathrm{s}$ in a single-mode single-core fiber reported in Ref. [29], it is expected to reach transmission capacities of about $10 \mathrm{Pbit} / \mathrm{s}$ in FM-MFC transmission with more than 100 spatial channels using both the $\mathrm{C}$ - and the L-band. Considering these limitations on the transmission capacity, recently, a new approach was demonstrated to reach 10.16 Pbits/s using an ultra-dense SDM transmission with 114 spatial multiplicities using C+L-band 739 WDM 12 Gbaud dual-polarization (DP)-64-quadrature amplitude modulation (QAM)/16-QAM signals over $11.3 \mathrm{~km}$ employing a 6-mode-19-core fiber with lower differential mode delay and lower fiber attenuation in the $\mathrm{C}+\mathrm{L}$ band. Thus, Fig. 11 illustrates the 6-mode 10-core fiber shape fabrication and its transmission characteristics.

Even more, Fig. 12 shows the experimental configuration implemented to achieve SDM/WDM transmission of 10.16 $\mathrm{Pbit} / \mathrm{s}$ using $\mathrm{C}+\mathrm{L}-$ band. Using this configuration, a record transmission capacity of $10.16 \mathrm{Pbits} / \mathrm{s}$, and a spectral efficiency of $1099.9 \mathrm{bit} / \mathrm{s} / \mathrm{Hz}$ were reached using 64- QAM/16QAM signals and the $6 \mathrm{M}-19 \mathrm{CF}$ by assuming rate-adaptive forward error correction (FEC).

\section{Conclusion}

We have shown a review of the most recent research about SDM/WDM techniques. This work was composed of two sections. The first section was focused on showing the new contributions in the areas of PL, MCF, and FMF for their application in WDM/SDM. The second section presented works related to transmission rates using WDM and SDM multiplexing techniques as well as the integration of components such as PL, MCF, and FMF. Researches about these topics have allowed the combination of multiplexing techniques 
such as SDM and WDM. As a consequence, currently, it is possible to reach the rate of Petabits per second in data transmission. In conclusion, we consider SDM/WDM techniques will have to evolve and adapt to current user requirements for the delivery of services as internet, video on demand, data transfer, etc.

\section{Acknowledgments}

J. R. Warnes Lora, B. Tshibangu-Mbuebue, L. J. QuinteroRodríguez wish to thank the CONACyT for the student scholarships numbers 702299, 846218 and 465594, respectively.
1. T. Mizuno and Y. Miyamoto, High-capacity dense space division multiplexing transmission, Opt. Fiber Technol. 35 (2017) 108. doi:10.1016/j.yofte.2016.09.015.

2. G. Li, N. Bai, N. Zhao, and C. Xia, Space-division multiplexing: the next frontier in optical communication, Adv. Opt. Photonics 6 (2014) 413. doi:10.1364/aop.6.000413.

3. N. K. Fontaine, R. Ryf, J. Bland-Hawthorn, and S. G. LeonSaval, Geometric requirements for photonic lanterns in space division multiplexing, Opt. Express, 20 (2012) 27123. doi: $10.1364 / \mathrm{OE} .20 .027123$.

4. S. G. Leon-Saval, A. Argyros, and J. Bland-Hawthorn, Photonic lanterns, Nanophotonics 2 (2013) 429. doi : 10 .1515/ nanoph-2013-0035.

5. B. Ercan, R. Ryf, J. Bland-Hawthorn, J. R. S. Gil, S. G. Leon-Saval, and N. K. Fontaine, Mode-selective dissimilar fiber photonic-lantern spatial multiplexers for few-mode fiber, 39th Eur. Conf. Exhib. Opt. Commun. (2013) 1221. doi:10. $1049 / \mathrm{cp} .2013 .1680$.

6. S. Yerolatsitis, I. Gris-Sánchez, and T. A. Birks, Adiabaticallytapered fiber mode multiplexers, Opt. Express 22 (2014) 608. doi:10.1364/OE.22.000608.

7. T. A. Birks, I. Gris-Sánchez, and S. Yerolatsitis, The photonic lantern, Opt. InfoBase Conf. Pap., 167 (2014) 107. doi: 10.1364/cleo_si.2014.sm2n.3.

8. S. G. Leon-Saval, N. K. Fontaine, J. R. Salazar-Gil, B. Ercan, R. Ryf, and J. Bland-Hawthorn, Mode-selective photonic lanterns for space-division multiplexing, Opt. Express 22 (2014) 1036. doi:10.1364/OE.22.001036.

9. A. M. Velázquez-Benítez et al., Scaling photonic lanterns for space-division multiplexing, Sci. Rep., 8 (2018) 1. doi: 10. 1038/s41598-018-27072-2

10. S. G. Leon-Saval, T. A. Birks, J. Bland-Hawthorn, and M. Englund, Multimode fiber devices with single-mode performance, Opt. Lett., 30 (2005) 2545. doi:10.1364/OL. 30. 002545 .

11. S. G. Leon-saval, Multimode Photonics, Optical Transition Devices for Multimode Control, no. July, (2014) pp. 95. https : //doi.org/10.1117/12.2231924

12. J. von Hoyningen-Huene, R. Ryf, and P. Winzer, LCoS-based mode shaper for few-mode fiber, Opt. Express 21 (2013) 18097. doi:10.1364/OE.21.018097.

13. Z. Tan et al., High Speed Band-Limited 850-nm VCSEL Link Based on Time-Domain Interference Elimination, IEEE Photonics Technol. Lett., 29 (2017) 751. doi:10.1109/LPT. 2017.2683526 .
14. X. Shen, J. M. Kahn, and M. A. Horowitz, by adaptive optics 30 (2005) 2985. https://doi.org/10.1364/OL.30. 002985

15. D. H. Sim, Y. Takushima, and Y. C. Chung, High-speed multimode fiber transmission by using mode-field matched centerlaunching technique, J. Light. Technol. 27 (2009) 1018. doi: 10.1109/JLT.2008.2005040

16. L. Ma, N. Hanzawa, K. Tsujikawa, and Y. Azuma, Launch device using endlessly single-mode PCF for ultra-wideband WDM transmission in graded-index multi-mode fiber, Opt. Express 20 (2012) 24903. doi:10.1364/oe.20.024903

17. J. Yu et al., Weakly-coupled mode division multiplexing over conventional multi-mode fiber with intensity modulation and direct detection, Front. Optoelectron. 12 (2019) doi:10. 1007/s12200-018-0834-9.

18. M. Wada et al., High density few-mode multicore fibre amplifier for energy efficient SDM transmission, IET Conf. Publ., 2019 (2019) 6 doi:10.1049/cp.2019.0918

19. E. Le Taillandier De Gabory, K. Matsumoto, and H. Takeshita, Advances in Power-Efficient SDM Transmission Technologies, Asia Commun. Photonics Conf. ACP 2018 (2018) 2018. doi : $10.1109 / \mathrm{ACP} .2018 .8596122$

20. G. Rademacher et al., Long-Haul Transmission over Few-Mode Fibers with Space-Division Multiplexing, J. Light. Technol. 36 (2018) 1382. doi:10.1109/JLT.2017.2786671

21. H. Hu et al., Ultrahigh-Spectral-Efficiency WDM/SDM Transmission Using PDM-1024-QAM Probabilistic Shaping with Adaptive Rate, J. Light. Technol. 36 (2018) 1304. doi: 10. $1109 /$ JLT.2017.2787340.

22. J. C. Alvarado-Zacarias et al., 7-core X 6-mode photonic lantern mode multiplexer, IET Conf. Publ 2019 (2019) 4. do i : $10.1049 / \mathrm{cp} .2019 .0919$.

23. J. Cui et al., A High-selectivity Photonic Lantern Demultiplexer for Weakly-coupled Mode Group Demultiplexing over MMF, 2019 18th Int. Conf. Opt. Commun. Networks (2019) 1. doi:10.1109/ICOCN.2019.8934086.

24. J. R. Warnes-Lora, I. E. Zaldivar-Huerta, J. RodriguezAsomoza, R. Amezcua-Correa, J. E. Antonio-López, and A. García-Juárez, Performance Evaluation of a Photonic Lantern by Transmitting Microwave Signals, 2018 Photonics North 10 (2013) 2018. doi:10.1109/PN.2018.8438830.

25. C. Castro et al., 15 x 200 Gbit/s 16-QAM SDM Transmission over an Integrated 7-Core Cladding-Pumped Repeatered Multicore Link in a Recirculating Loop, J. Light. Technol. 36 (2018) 349. doi:10.1109/JLT.2017.2741495.

26. F. Bao, T. Morioka, L. K. Oxenløwe, and H. $\mathrm{Hu}, 300 \mathrm{~Gb} / \mathrm{s}$ IM/DD based SDM-WDM-PON with laserless ONUs, Opt. Express, 26 (2018) 7949. doi:10.1364/oe.26.007949. 
27. N. P. Diamantopoulos et al., 400-Gb/s DMT-SDM transmission based on membrane DML-array-on-silicon, J. Light. Technol. 37 (2019) 1805.doi:10.1109/JLT.2018.2885792.

28. D. Soma, Y. Wakayama, S. Beppu, S. Sumita, T. Tsuritani, and P. Paper, 10.16-Peta-B/s Dense SDM / WDM Transmission
Over 6-Mode 19-Core Fiber Across the C + L Band, 36 (2018) 1362.https://doi.org/10.1364/JLT.36.001362

29. D. Qian, E. Ip, M. Huang, M. Li, A. Dogariu, and S. Zhang, 1. 05Pb / s Transmission with $109 \mathrm{~b} / \mathrm{s} / \mathrm{Hz}$ Spectral Efficiency using Hybrid Single- and Few-Mode Cores, pp. 5. https: //doi.org/10.1364/FIO.2012.FW6C.3 